\title{
Molecular detection and characterization of a 16Srll-D phytoplasma associated with streak yellows of date palm in Oman
}

\author{
Chamran Hemmati $^{1,2} \cdot$ Ali M. Al-Subhi ${ }^{1} \cdot$ Mohammed T. Al-Housni $^{1}$ • Abdullah M. Al-Sadi ${ }^{1}$ (D)
}

Received: 3 June 2020 / Accepted: 1 September 2020 / Published online: 9 September 2020

(C) Australasian Plant Pathology Society Inc. 2020

\begin{abstract}
Date palm is the most important fruit crop in Oman, covering about 50\% of the agricultural area in the country. During a survey in 2018-2020, symptoms resembling phytoplasma diseases were observed in date palms in Muscat, Oman. Date palms showed streak yellows and dryness of young and mature leaflets and leaves. Polymerase chain reaction (PCR) assays were conducted using the universal primer pairs P1/P7 followed by R16F2n/R16r2n. In addition, the phytoplasma genes imp, secA, and tuf were also targeted in semi-nested PCR assays by using specific primers for each gene. Symptomatic trees were positive for phytoplasma presence in nested and semi-direct PCR, however, no amplification was observed from the symptomless and negative control. Sequence analysis of the rRNA, imp, secA, and tuf genes confirmed that the phytoplasma associated with streak yellow date palm (SYDP) is a strain of the 16SrII-D subgroup. This is the first record of association of a phytoplasmas of the 16SrII-D subgroup with date palms in Oman. This finding increased our knowledge about the distribution of phytoplasma diseases in date palms in the world.
\end{abstract}

Keywords Phoenix dactylifera $\cdot 16$ SrII-D $\cdot$ Multilocus $\cdot$ Immunodominant $\cdot$ Translocase $\cdot$ Translation elongation

Date palm (Phoenix dactylifera $\mathrm{L}$.) is the most important fruit crop in the arid regions of the Middle East. Based on FAO, date palm production totaled 8.5 Mt in 2018 (FAO 2019). In Oman, date palm occupies approximately $50 \%$ of the agricultural area, with a total production of 369,000 tons in 2018, placing Oman the 8th producer of dates in the world (FAO 2019).

Date palm production is affected by several diseases. Phytoplasmas are known to cause major diseases in palms, including the lethal yellowing disease (Gurr et al. 2016). Several reports are available on the association between diverse phytoplasma groups and date palms, including the $16 \mathrm{SrI}$ in Egypt (Alkhazindar 2014), 16SrII in Saudi Arabia (Alhudaib et al. 2007), 16SrIV in the USA (Ntushelo et al.

Electronic supplementary material The online version of this article (https://doi.org/10.1007/s13314-020-00404-w) contains supplementary material, which is available to authorized users.

Abdullah M. Al-Sadi

alsadi@squ.edu.om

1 Department of Plant Sciences, College of Agricultural and Marine Sciences, Sultan Qaboos University, Muscat, Oman

2 Department of Agriculture, Minab Higher Education Center, University of Hormozgan, Bandar Abbas, Iran; Plant Protection Research Group, University of Hormozgan, Bandar Abbas, Iran
2012), 16SrVI and VII in Iran (Zamharir and Eslahi 2019) and $16 \mathrm{Sr}$ XIV in Sudan (Cronjé et al. 2000). The most known symptoms included yellowing and streak yellows in leaves, while in some regions lethal yellowing symptoms have been observed.

During a survey in 2018-2020, date palms showing streak yellows and dryness of young and mature leaflets and leaves were observed in Muscat, Oman. The objective of the study was to determine the association of phytoplasmas with the symptoms.

A total of 25 date palm samples showing streak leaf yellowing and leaflet/leaf dryness (Fig. 1) were collected from Muscat during 2018-2020. The symptoms were apparent on trees, 5-20-year-old over all seasons. Ten symptomless date palms, seemingly healthy, were included for comparative purposes. Samples were obtained from leaves, trunk, and roots. Wood from basal trunk was sampled by using a portable electric drill and roots near the soil surface were collected by shovel. Samples were put in a plastic bag, labeled, and transferred to Sultan Qaboos University for further processing.

Total nucleic acid was extracted from leaf tissue, woods and roots by using a procedure described by Doyle and Doyle (1990) for the leaves and Harrison et al. (2008) for the trunk and root samples. A nested-PCR assay primed by $\mathrm{P} 1 / \mathrm{P} 7$, followed by $\mathrm{R} 16 \mathrm{~F} 2 \mathrm{n} / \mathrm{R} 16 \mathrm{R} 2 \mathrm{n}$ was conducted to 
Fig. 1 Symptoms of phytoplasma infection in date palms: yellow streaking (A, B), premature dryness of leaflets (B)

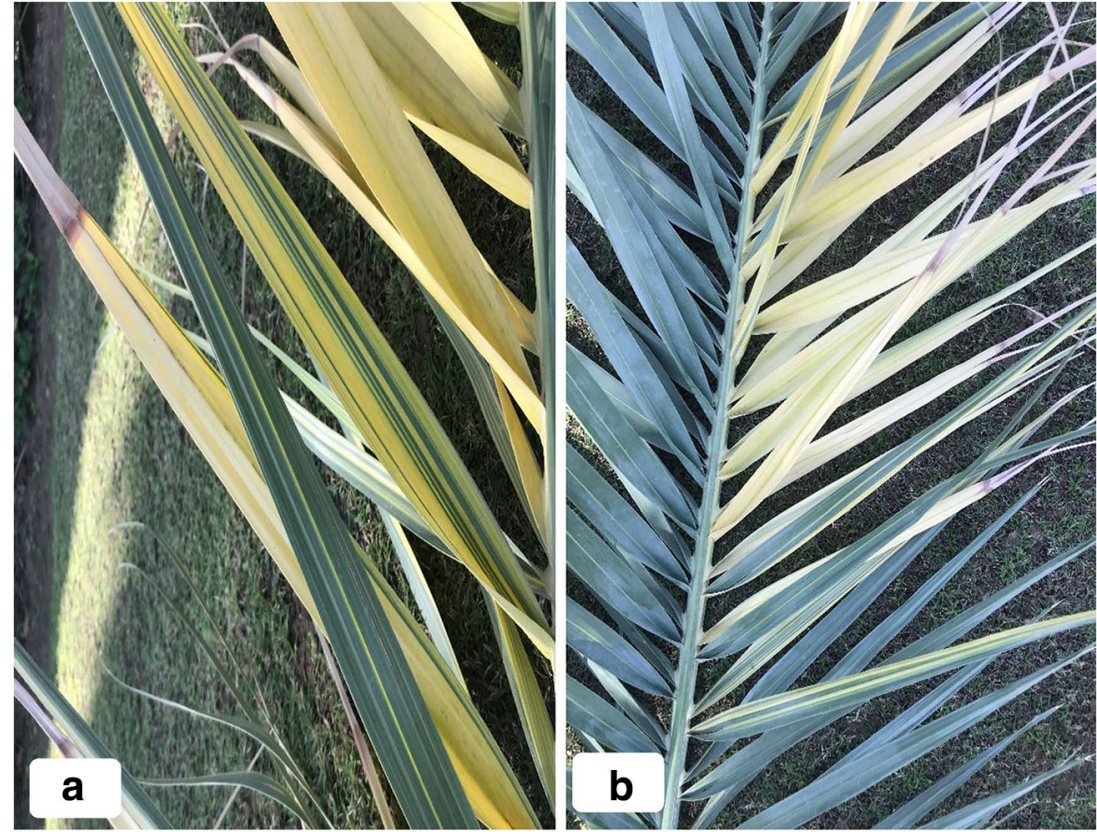

amplify the 16S rRNA region (Deng and Hiruki 1991; Gundersen and Lee 1996). In addition, distilled water (DW) and DNA from symptomless trees were used as negative control, while alfalfa and lime witches' broom phytoplasmas were used as positive control (Al-Subhi et al. 2018).

The tuf, imp and secA genes were amplified using primer pairs specific for phytoplasmas in the 16SrII group. The TUFII-F1 and TUF-II-R1 were utilized to prime the elongation factor TU targeting $1490 \mathrm{bp}$, followed by using the seminested PCR primers TUF-II-F2 and TUF-II-R1, which target $1094 \mathrm{bp}$ of the tuf gene. The imp gene was amplified by using IMP-II-F1 and IMP-II-R1 primer pairs (targeting (786 bp) in the direct PCR followed by IMP-II-F1/IMP-II-R1 (targeting $717 \mathrm{bp}$ ) in the semi-nested PCR assay (Al-Subhi et al. 2018). A partial of translocase protein A gene was amplified in semidirect PCR assays by using SecA-II-F1/SecA-II-R1 in the first reaction followed SecA-II-F1/SecAII-R4 in the second reaction. The PCR reaction mix and conditions of the $16 \mathrm{~S}$ rRNA, tuf, imp and secA were as described by Al-Subhi et al. (2018).

Three positive products of each gene were sent to Macrogen (South Korea) and directly sequenced by the primer pairs used in the nested and semi-nested assays. Genious Prime (version 2020.1.1) (Kearse et al. 2012) and ClustalX were utilized to assemble and align the obtained sequences. A neighbor-joining method using Kimura-parameter models with 1000 replicates for bootstrap analysis were used to construct phylogenetic tree by MEGA 6 software (Tamura et al. 2013). To root the phylogenetic tree of $16 \mathrm{~S}$ rRNA, tuf, and secA, B. subtilis reference sequences AB042061, D10279, and GCA_000789275 were used, respectively. Reference phytoplasma sequences of the four genes from NCBI
GenBank were included in phylogenetic tree construction (Supplementary Tables 1,2). A combined tree based on the four genes was created with MEGA 6 using the model and method described in single gene tree.

Symptomatic date palms showed longitudinal streak yellows in the midribs and leaflets, which developed on other parts of the leaves. This was followed by dryness of the yellow leaflets from the top to the bottom. In addition, some palms displayed dryness of the older leaves and some young leaves.

In total, 12 leaf samples showing streak yellows and leaf dryness were positive in PCR assays of the 16S rRNA but no amplification was observed from the trunk and root samples. PCR amplification of the symptomatic plants and positive control showed the expected size products in semi-nested PCR assays, which were 1250, 726, 1320, and $998 \mathrm{bp}$ for the 16S rRNA, imp, secA, and tuf genes, respectively. No fragments were amplified in DW and healthy plants.

Sequence analysis showed that the three sequences of the 16S rRNA had $100 \%$ identity to each other and $100 \%$ sequence identity with those of Cycas revoluta lethal yellowing phytoplasma CLY clones (MT293343-6) from Oman, Roystonea regia yellowing phytoplasma clones (MT232233-6) from Oman, and Alfalfa phytoplasma clone AP2 (KY449416) from Sudan. Neighbor-joining phylogenetic tree analyses of the partial 16S rRNA sequences (1152 bp) indicated that date palm streak yellow (DPSY) phytoplasma clustered in a subclade with phytoplasma group II-D with $100 \%$ bootstrap support. They were closely related to Alfalfa witches' broom (KX35856), and "Candidatus Phytoplasma australasia" (Y10097), which are from the 16SrII-D subgroup phytoplasma (Fig. 2A). 


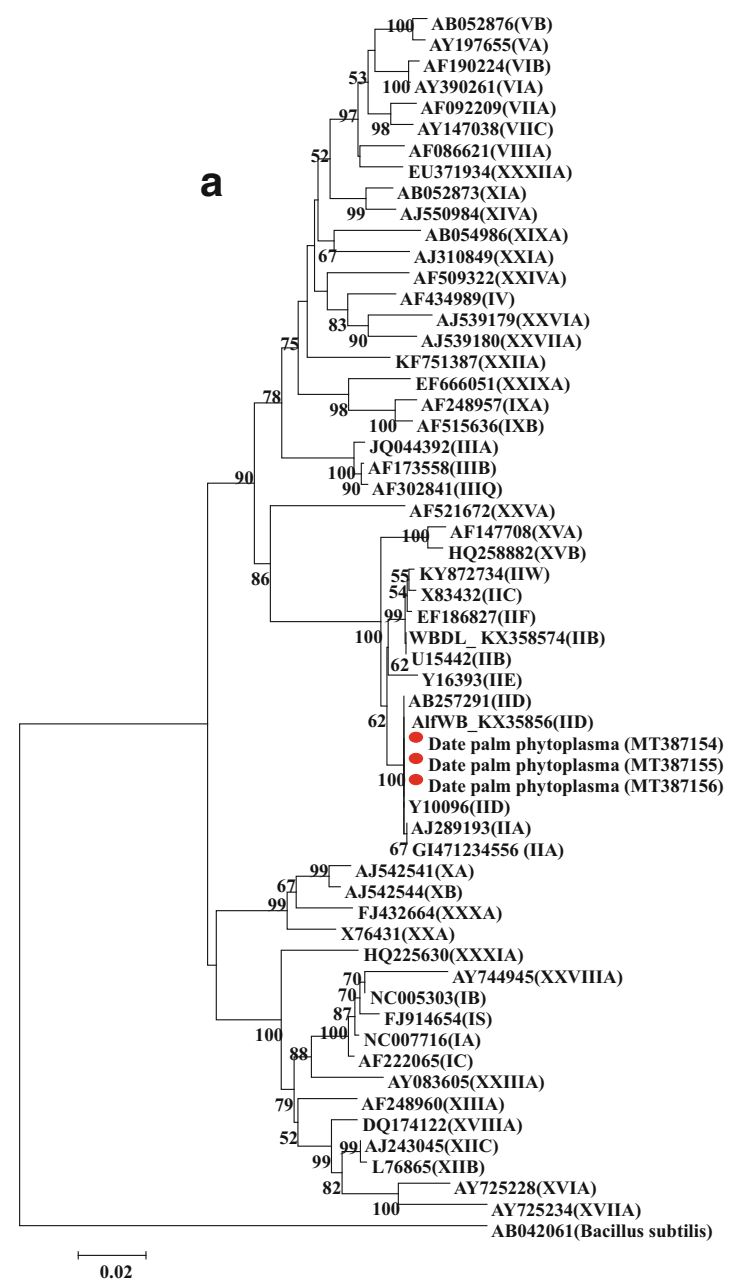

Fig. 2 Phylogenetic tree based on the 16S ribosomal RNA gene sequences (A) and the combined 16S ribosomal RNA, tuf, secA and imp gene sequences (B) of date palm phytoplasmas (black circles) with 41 published phytoplasma sequences from GenBank. The tree was rooted

The imp gene sequence analysis using BLASTn search showed $100 \%$ identity with Vicia faba phyllody (KX358606), Cucurbita pepo phytoplasma (KX358601), and Medicago sativa phytoplasma isolate (KX358600), which are from Oman. The three sequences clustered with tomato big bud (JQ745285) and alfalfa witches' broom phytoplasmas, which are strains of the 16SrII-D subgroup (Supplementary Fig. 1A).

The secA gene of DPSY phytoplasma showed $100 \%$ identity with Cicer arietinum phyllody (KX358585), Solanum melongena big bud (KX358584), and Pisum aestivum phyllody phytoplasma (KX358583), which are from Oman. A phylogenetic tree constructed using $536 \mathrm{bp}$ from the $\sec \mathrm{A}$ sequences and 32 other phytoplasamas showed that the three sequences clustered with tomato big bud (KJ462021) and alfalfa witches' broom (KX358576) phytopalsmas, which are strains of the 16SrII-D (Supplementary Fig. 1B).

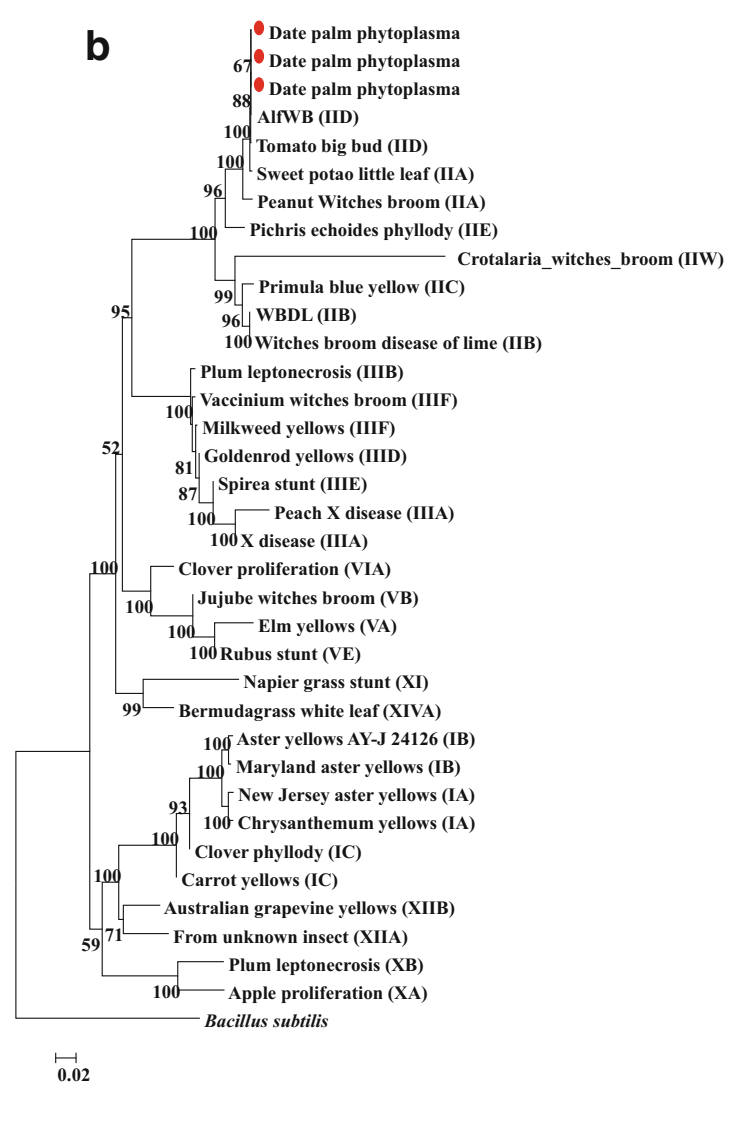

using Bacillus subtilis (AB04261, GCA000789275). The phylogenetic tree was constructed by the neighbor-joining method and units are the number of base substitutions per site. Bootstrap values are expressed as percentage of 1000 replicates

The BLASTn algorithms against the GenBank for the tuf gene showed the highest identity $99.8 \%$ (995 identical bases) with Solanum melongena big bud (KX358596), Pisum sativum phyllody (KX358595), and Vicia faba phyllody (KX358594) from Oman. The phylogenetic tree based on 385 bp of tuf sequences of DPSY and 41 other phytoplasma from GenBank is shown in Supplementary Fig. 1C. The tuf tree placed three $t u f$ sequences of SYDP phytoplasma within one clade of the 16SrII-D phytoplasmas with alfalfa witches' broom (KX358588) and sweet potato little leaf (JQ824250) (16SrII-D), with $100 \%$ bootstrap support.

Three sequences for each gene were deposited in GenBank database under the accession numbers (MT387154, MT387155, MT387156) for the 16S rRNA, (MT361012, MT361013, MT361014) for the imp, (MT361006, MT361007, MT361008) for the secA, and (MT361009, MT361010, MT361011) for the tuf genes. 
The combined tree placed the DPSY phytoplasma with the 16SrII-D clade, with a high bootstrap support (88\%) (Fig. 2B).

In this study, the associated phytoplasma with streak yellow date palms was well characterized using multiple gene sequencing. Diverse phytoplasma groups have been reported associated with different symptoms in date palms. For example, Harrison et al. (2008) reported the association between $P$. dactylifera and 16SrIV-D subgroups and a mix infection of 16SrIV-A and IV-F showing severe symptoms like falling of premature fruits, yellowing and browning the leaves, dryness of the new leaves and finally death of the trees in the USA. Similar symptoms have also been observed in date palms associated with 16SrIV-A in Kuwait (AlAwadhi et al. 2002) and Tanzania (Tymon et al. 1998). Al-Wijam disease showing reduction in fruits and stalk size, streak yellows in leaves and fruits, and leaf stunting has been reported in association with 16SrI and 16SrII-D subgroup phytoplasmas (Alhudaib et al. 2007; Alhudaib et al. 2008). White tip die-back displaying severe chlorosis of the new leaves, white longitudinal streaks of the midribs, and drying/ whiteness of the crown has been reported to be associated with 16SrXIV in Sudan (Cronjé et al. 2000). However, the characteristic streak yellowing of leaves has been reported in Iran (Zamharir and Eslahi 2019). This is the first report of association of 16SrII-D with date palm in Oman. Strains of $16 \mathrm{Sr}$ II-D phytoplasma subgroups have been reported in association with diverse plant species in Oman (Al-Subhi et al. 2018). Our results obtained from phylogenetic tree of the three genes was in accordance with 16S rRNA analysis, and the combined phylogenetic tree confirmed the association between DPSY and a phytoplasma of the 16SrII-D subgroup.

The present study reports the first observation of 16SrII-D phytoplasmas associated with streak yellow of date palm in Oman. In addition, this study increased our knowledge of the geographical distribution of phytoplasma diseases of date palms in the world. 16SrII-D phytoplasma strains are the most dominant phytoplasmas types and have widespread host species in Oman. Further studies are needed to investigate the insect vectors of this phytoplasma in Oman.

Acknowledgments Thanks to Sultan Qaboos University for funding the study (SR/AGR/CROP/17/01 and RF/AGR/CROP/19/03).

\section{Compliance with ethical standards}

Conflict of interest The authors declare no conflict of interest. All authors fully agree for submission of the manuscript.

Ethical approval The study does not contain any studies with human participants or animals performed by any of the authors.

\section{References}

Al-Awadhi HA, Hanif A, Suleman P, Montasser MS (2002) Molecular and microscopical detection of phytoplasma associated with yellowing disease of date palms Phoenix dactylifera L. in Kuwait. Kuwait J Sci Eng 29:87-109

Al-Subhi AM, Hogenhout SA, Al-Sadi AM, Al-Yahyai RA (2018) Detection, identification, and molecular characterization of the 16SrII-D phytoplasmas infecting vegetable and field crops in Oman. Plant Dis 102:576-588. https://doi.org/10.1094/PDIS-0717-0935-RE

Alhudaib K, Arocha Y, Wilson M, Jones P (2007) Al-Wijam, a new Phytoplasma disease of date palm in Saudi Arabia. Bull Insectol 60:285-286

Alhudaib K, Arocha Y, Wilson M, Jones P (2008) First report of a 16SrI, Candidatus Phytoplasma asteris group phytoplasma associated with a date palm disease in Saudi Arabia. Plant Pathol 57:366. https://doi. org/10.1111/j.1365-3059.2007.01667.x

Alkhazindar M (2014) Detection and molecular identification of aster yellows phytoplasma in date palm in Egypt. J Phytopathol 162: 621-625. https://doi.org/10.1111/jph.12241

Cronjé P, Dabek AJ, Jones P, Tymon AM (2000) First report of a phytoplasma associated with a disease of date palms in North Africa. Plant Pathol 49:801. https://doi.org/10.1046/j.1365-3059.2000. 00504.x

Deng S, Hiruki C (1991) Amplification of 16S rRNA genes from culturable and non-culturable mollicutes. J Microbiol Methods 14: $53-61$

Doyle J, Doyle JL (1990) Isolation of plant DNA from fresh tissue. Focus 12:13-15

FAO (2019) FAOSTAT. FAO. http://www.fao.org/faostat/en/\#data/QC/ visualize. 8/12/2019

Gundersen DE, Lee IM (1996) Ultrasensitive detection of Phytoplasmas by nested PCR assays using two universal primer pairs. Phytopathol Mediterr 35:144-151

Gurr GM, Johnson AC, Ash GJ, Wilson BAL, Ero MM, Pilotti CA, Dewhurst CF, You MS (2016) Coconut lethal yellowing diseases: a phytoplasma threat to palms of global economic and social significance. Front Plant Sci 7:1521. https://doi.org/10.3389/fpls.2016. 01521

Harrison NA, Helmick EE, Elliott ML (2008) Lethal yellowing-type diseases of palms associated with phytoplasmas newly identified in Florida, USA. Ann Appl Biol 153:85-94. https://doi.org/10.1111/ j.1744-7348.2008.00240.x

Kearse M, Moir R, Wilson A, Stones-Havas S, Cheung M, Sturrock S, Buxton S, Cooper A, Markowitz S, Duran C, Thierer T, Ashton B, Meintjes P, Drummond A (2012) Geneious basic: an integrated and extendable desktop software platform for the organization and analysis of sequence data. Bioinformatics 28:1647-1649

Ntushelo K, Harrison NA, Elliott ML (2012) Comparison of the ribosomal RNA operon from Texas Phoenix decline and lethal yellowing phytoplasmas. Eur J Plant Pathol 133:779-782. https://doi.org/10. 1007/s10658-012-0007-4

Tamura K, Stecher G, Peterson D, Filipski A, Kumar S (2013) MEGA6: molecular evolutionary genetics analysis version 6.0. Mol Biol Evol 30:2725-2729

Tymon AM, Jones P, Harrison NA (1998) Phylogenetic relationships of coconut phytoplasmas and the development of specific oligonucleotide PCR primers. Ann Appl Biol 132:437-452. https://doi.org/10. 1111/j.1744-7348.1998.tb05220.x

Zamharir MG, Eslahi MR (2019) Molecular study of two distinct phytoplasma species associated with streak yellows of date palm in Iran. J Phytopathol 167:19-25. https://doi.org/10.1111/jph.12769 